\title{
A New Quantitative Metric for Liver Classification from Ultrasound Images
}

\author{
Mandeep Singh, S. Singh, and S. Gupta, Member, IACSIT
}

\begin{abstract}
This paper presents a new quantitative metric which can be used as indirect method for characterization of liver from ultrasound images. This new metric is inspired from the visual criterion considered by the radiologists through texture and echogenicity of the Liver in ultrasound image. The proposed metric is a single parameter extracted from 6 texture features on the basis of Homogeneity, Roughness, Contrast, Granularity and Orientation of the liver surface for classification into Fatty or Normal liver. The main contribution of the present work is that the best texture features are selected on the basis of Fisher's linear discriminative analysis and then formulated into a single metric by considering their weightage similar to the visual criterion used for liver classification.
\end{abstract}

Index Terms-Echogenicity, liver classification, steatosis, texture analysis.

\section{INTRODUCTION}

Ultrasound is an imaging modality that has become widely popular because of its ability to visualize many human soft tissues without any harmful effect. Since ultrasound images suffer from speckle noise, thus it is difficult to differentiate an organ towards its specific pathological changes. However, the granular structure of the tissue or area can be studied and analysed to characterize it. This specific granular pattern of normal liver and fatty liver can be described as texture and thus Texture analysis for tissue characterization may be used to study and correlate the physiological changes in the liver. Moreover this approach may provide some important information that may not be obtained through visual interpretation of ultrasound images. The Radiologists distinguish between normal and fatty liver by using five major parameters through visual interpretations. These are Contrast, Homogeneity, Roughness, ratio of Echogenicity of Liver and Kidney, Size and the overlapping of Liver over neighbouring organs. In the present study an attempt is made to propose a quantitative index using some selected texture features to assist the radiologist.

In the past few years, many statistical features which describe the physical properties of the tissue have been

Manuscript received June 16, 2012; revised July 27, 2012. This work is partially supported by the project under SERC Fast-track scheme no. SR/FTP/ETA-065/2008 funded by Department of science and technology (Govt. of INDIA) New Delhi. Authors are thankful to radiologists from PGIMER Chandigarh and Delta Diagnostic Centre Patiala for their valuable help in this work.

Mandeep Singh is with the Thapar University, Patiala India (e-mail: mdsingh@ thapar.edu).

Sukhwinder Singhis Professor and Head at CSED, UIET, Panjab University, and Chandigarh, India (e-mail: sukhdalip@yahoo.com).

S Gupta is Professor CSED, UIET, Panjab University, and Chandigarh, INDIA (e-mail: savita2k8@yahoo.com). published to distinguish between normal and abnormal ultrasonic liver images [1], [2]. The most commonly used texture features that have been applied successfully to real-world textures are the spatial gray-level dependence matrices (SGLDM) by Haralick [3], the Fourier power spectrum (FPS) [4], the Laws' texture energy measures (TEM) [5]. The fractal concept developed by Mandelbrot [6] provides an excellent representation of the roughness of natural surfaces. To evaluate the liver diseases using Ultrasound parameters like change in echo-texture, echogenicity, liver surface, Inferior edge etc. was first proposed by Nicolau et. al.[7]. Then Vehmas et. al. measured the echogenicity ratio of Liver to that of Kidney as an indirect metric to diagnose the steatosis [8]. Recently Webb et. al. have proposed an approach to quantify the Liver steatosis (fat infiltration) using computerized Hepto-renal Index [9].

\section{A. Liver Physiology and Characteristics}

Liver pathologies can be classified into two main categories according to the degree of dispersion of the disease. The first category is the localized liver diseases in which the pathology is concentrated in small spot(s) in one or both of the liver lobes while the rest of the liver tissue remains normal. The second category is the diffused liver diseases in which at least one complete lobe of the liver is affected by the disease. Steatosis (or fatty liver) is the second category disease in which fat is infiltrated in the Liver. A visual criterion for diagnosing a fatty liver is done from 'bright liver', which is evaluated by comparing the liver echogenicity to that of right kidney cortex at the same depth [10].

\section{MAterials AND Methodology}

\section{A. Image Acquisition}

All images are acquired with GE Medicare's Voluson730 PRO machine by Curved array transducer probe. A range of 3-3.6 MHz frequency is used to acquire the images of the object. Following criteria is adopted throughout the analysis.

1) ROI location: To avoid the distorting effects in ultrasonic wave patterns, such as side lobes and grating lobes, the region of interest (ROI) is selected each time along the center line of the image.

2) ROI Size and shape: To get reliable analysis results, the number of pixels in the ROI must be at least 800 to provide the reliable statistics [11]. Keeping this criterion, a square size of 30x30 pixels (containing 900 pixels) is selected.

3) Fasting condition of the patient: It has been suggested that patients should be fasting for eight hours before any 
scan to avoid the effects of changing the liver glycogen and water storage on ultrasound attenuation [12].

\section{B. Texture features}

Liver surface can be treated as a texture and thus various texture models are selected to analyze the liver ultrasound images. The main parameters those are considered subjectively are echogenicity (brightness), contrast, homogeneity, granularity and smoothness of the liver surface. But in this work some objective features are used to measure the above parameters. To increase the feature space, five different texture models are used; Spatial gray-level dependence matrices (SGLCM), Statistical Feature Matrix (SFM), Law's Texture energy measure (TEM), Fourier power spectrum (FPS) and Fractal features.

a) SGLCM: The Spatial Grey Level Co-occurrence Matrix SGLCM texture features, as proposed by Haralick et al. [3], are the most frequently used texture features. Based on the probability density functions, 14 features are studied.

b) Laws' Texture Energy Measures: In this study, vectors of length $1=7, \mathrm{~L}=(1,6,15,20,15,6,1), \mathrm{E}=(-1,-4,-5,0$, $5,4,1)$, and $\mathrm{S}=(-1,-2,1,4,1,-2,-1)$ are used. The following 6 TEM features are calculated: LL Texture Energy, EE, SS, LE average texture energy from LE and EL kernels, ES average texture energy from ES and SE kernels, and LS average from LS and SL kernels [5].

c) Statistical Feature Matrix: The statistical-feature matrix (SFM) measures the statistical properties of pixel pairs. Based on the SFM, the following 3 texture features are computed: Coarseness, Periodicity and Roughness [13].

d) Fourier Power Spectrum: FPS contains information on the texture orientation, grain size, and texture contrast of the image. The Radial sum $\mathrm{F}_{r}$ and the Angular sum $\mathrm{F}_{a}$ of the discrete Fourier transform are computed to describe texture [14].

e) Fractal feature: The Hurst coefficients $\mathrm{H}_{1}$ and $\mathrm{H}_{2}$ are computed for different image resolutions. The fractal dimension can measure the roughness and the granular structure of an image [13].

\section{Feature Selection and Reduction}

Five different texture models are used to extract 27 texture features from each ROI. For each image all features are calculated, and finally their mean and standard deviation is calculated among the one class for a given feature. Since the pathological results were available, so linear discriminative analysis using Fisher's discriminative ratio (FDR) is calculated for each feature using the equation 1 [15].

$$
F D R=\left|\frac{m_{1}-m_{2}}{\sqrt{\left(\sigma_{1}^{2}+\sigma_{2}^{2}\right)}}\right|
$$

where $m_{1}$ anu in 2 anc numans anu $v_{1}$ and $\sigma_{2}$ are the standard deviations of fatty and normal liver respectively. High value of FDR signifies the higher ability of a feature to classify the image into different classes.

\section{RESULTS AND DiSCUSSION}

All the Normal and fatty liver images are analyzed quantitatively for 27 texture parameter values. Then their class-wise mean and standard deviation is reported and FDR is calculated. Finally the highly correlated features are removed and out of the remaining features, only those features are selected which has the high relevance with 'Echogenicity' and surface smoothness being evaluated by radiologist subjectively. These features are given in Table I. From the Table I it is evident that the feature which has high interclass separation ability (high FDR), they are related with the parameters like echo-texture (IDM or homogeneity and $H_{l}$ ), Contrast and smoothness of liver surface (Roughness, $F_{a}$ and $\left.H_{l}\right)$. Similarly, the Difference of Entropy is related with the liver surface.

TABLE I: BEST SIX FEATURES

\begin{tabular}{|c|c|c|c|c|c|c|}
\hline \multirow[b]{2}{*}{ Feature } & \multirow{2}{*}{$\begin{array}{l}\text { Visual } \\
\text { meaning }\end{array}$} & \multicolumn{2}{|c|}{ Fatty } & \multicolumn{2}{|c|}{ Normal } & \multirow[b]{2}{*}{ FDR } \\
\hline & & MEAN & \begin{tabular}{|c|} 
STDE \\
$\mathrm{V}$ \\
\end{tabular} & MEAN & $\begin{array}{c}\text { STDE } \\
\mathrm{V}\end{array}$ & \\
\hline $\begin{array}{c}\text { Diff_Entro } \\
\text { py }\end{array}$ & $\begin{array}{l}\text { Inverse of } \\
\text { homogeneity }\end{array}$ & 0.0894 & 0.0143 & 0.1891 & 0.0228 & 3.7044 \\
\hline IDM & Homogeneity & 0.9754 & 0.0072 & 0.9193 & 0.0167 & 3.0847 \\
\hline Contrast & Contrast & 0.0673 & 0.017 & 0.1667 & 0.0345 & 2.5844 \\
\hline $\mathrm{H} 1$ & Smoothness & 0.4023 & 0.0208 & 0.4535 & 0.021 & 1.7322 \\
\hline $\mathrm{F}_{\mathrm{a}}$ & Orientation & 1934.76 & $\begin{array}{c}1002.3 \\
4\end{array}$ & 2943.18 & 529.76 & 0.8894 \\
\hline $\begin{array}{c}\text { Rough_ } \\
\mathrm{S}\end{array}$ & Roughness & 2.4176 & 0.0425 & 2.3768 & 0.0371 & 0.7232 \\
\hline
\end{tabular}

\section{A. New Metric}

Each feature has different FDR and different weightage towards the final classification. So in the proposed metric, the value of each feature is measured by its FDR and its value. If the FDR value of feature $F_{1}$ is $F_{D R}$, and its value for a particular ROI is $\mathrm{X}_{1}$ (mean- present value/std. dev.), then the value of feature $F_{1}$ for class ' $a$ ' (Fatty Liver) can be $V_{a} 1$ as:

$$
V_{a} 1=F D R_{1} \times X_{1}
$$

And the total value of an image for class ' $a$ ' for all six features will be $V_{a}$

$$
V_{a}=V_{a} 1+V_{a} 2+V_{a} 3+V_{a} 4+V_{a} 5+V_{a} 6
$$

Similarly $V_{b}$ can be found for all 6 features in class ' $\mathrm{b}$ ' (Normal liver).

$$
V_{b}=V_{b} 1+V_{b} 2+V_{b} 3+V_{b} 4+V_{b} 5+V_{b} 6
$$

The new proposed metric $\mathbf{E}$ is defined as

$$
E=V_{a}-V_{b}
$$

If ' $E$ ' is greater than zero, then the image is of Fatty Liver and if $\mathrm{E}$ is negative, it is Normal liver. More the difference in $V_{a}$ and $V_{b}$, more the confidence level of result is there. If the value is close to zero then the radiologist has to investigate in more detail. So indirectly ' $E$ ' is a measure of echogenicity which the radiologists use to analyze the liver surface visually.

\section{B. Evaluation of the Metric}

Evaluation of the proposed metric is done on a separate set of 30 ultrasound images (15 Normal and 15 Fatty liver images). The results show that the proposed method has $92 \%$ 
accurate and $100 \%$ sensitive. As compared to a recently published work by Ribero and Sanches [16], where the reported accuracy is $95 \%$ and sensitivity is $100 \%$ using Bayesian classifier, however the proposed method is much simpler and faster. The only drawback of the proposed method is that their characterization criterion is machine dependent. Authors are working on more accurate and machine independent objective method which can be characterized the liver into more classes.

\section{CONCLUSION}

Texture analysis is very useful to discriminate fatty liver from normal liver images. After Statistical and Linear discriminating analysis, best 6 features are shortlisted with relevance to the visual criterion of echogenicity and echo-texture like Homogeneity, Contrast, Difference of entropy, Roughness etc. Finally a new metric ' $E$ ' is defined whose numerical value can be used to classify the Liver's state either Normal or Fatty. More the value of ' $E$ ' indicates more presence of steatosis. The evaluation results obtained from different 30 images reveal that the proposed method is $92 \%$ accurate and $100 \%$ sensitive. So this work proposes a quantitative metric based on texture analysis for the characterization of liver inspired from the visual criterion considered by the radiologists.

\section{ACKNOWLEDGMENT}

This work is partially supported by the project under SERC Fast-track scheme no. SR/FT/ETA-065/2008 funded by DST (Govt. of INDIA) New Delhi. Authors are thankful to radiologists from PGIMER Chandigarh and Delta Diagnostic Centre Patiala for their valuable help in this work.

\section{REFERENCES}

[1] R. Momenan, M. H. Loew, M. F. Insana, R. F. Wagner, and B. S. Garra, "Application of pattern recognition techniques in ultrasound tissue characterization," 10th Int. Cont Pattern Recognition, vol. 1, 1990, pp. 608-612.

[2] M. F. Insana, R. F. Wagner, B. S. Garra, R. Momenan, and T. H. Shanker, "Pattern recognition methods for optimizing multivariate tissue signatures in diagnostic ultrasound," Ultrasonic Imaging, vol. 8, 1986, pp. 165- 180.

[3] R. M. Haralick, K. Shanmugan, and H. Dinstein, "Texture features for image classification," IEEE Trans. Syst, Man, Cyber, vol. SMC-3, pp. 610-621, 1973.

[4] G. O. Lendaris and G. L. Stanley, "Diffraction pattern sampling for automatic pattern recognition," in Proc. IEEE, vol. 58, pp. 198-216, 1970.

[5] K. I. Laws, “Texture energy measures," in Proc. Image Understanding Workshop, pp. 47-51, 1979.

[6] B. B. Mandelbrot, the Fractal Geometry of Nature, San Francisco, CA, Freeman, 1982.

[7] C. Nicolau, L. Bianchi, R. Vilana, "Gray-scale ultrasound in hepatic cirrhosis and chronic hepatitis: diagnosis, screening, and intervention," Seminars in US, CT and MRI 2002, vol. 23, pp 3-18, 2002.

[8] T. Vehmas, A. Kaukiainen, K. Luoma, M. Lohman, M. Nurminen, and H. Taskinen, "Liver echogenicity: measurement or visual grading," Computerized Medical Imaging and Graphics vol. 28, pp 289-293, 2004.

[9] M. Webb, H. Yeshua, Z. S. E. Santo, Z. Halpern, and R. Oren, "Diagnostic value of a computerized Hepatorenal Index for Sonographic quantification of Liver Steatosis," American Journal of Radiology AJR:192, pp. 909 - 914, April 2009.

[10] J. P. McGahan and B. B. Goldberg, "Diagnostic Ultrasound," $2^{\text {nd }} e d$, Informa Healthcare, NY 2008.

[11] Y. M. Kadah, , A. A. Farag, J. M. Zurada, A. M. Badawi, and A. M. Youssef, "Classification Algorithms for Quantitative Tissue. Characterization of Diffuse Liver Disease from Ultrasound Images," IEEE Transactions on Medical Imaging, vol. 15, no. 4, 1996.

[12] T. A. Tuthill, R. B. Baggs, and K. J. Parker, "Liver Glycogen and Water Storage: Effect on Ultrasound Attenuation," Ultrasound in Medicine and Biology, vol. 15, no. 7, pp. 621-627, 1989.

[13] C. M. Wu, Y. C. Chen, and K. S. Hsieh, "Texture Features for Classification of Ultrasonic Liver Images," IEEE Transactions on Medical Imaging, vol. 11, no. 2, June 1992.

[14] I. M. Bankman, "Handbook of Medical Image Processing and Analysis," Academic Press, NewYork 2009.

[15] S. Theodoridis and K. Koutroumbas, "Pattern Recognition," 4th ed, Academic Press, 2008.

[16] R. Ribeiro and J. Sanches, "Fatty Liver Characterization and Classification by Ultrasound," Pattern Recognition and Image Analysis. H. Araujo, A. Mendonça, A. Pinho and M. Torres, Springer Berlin LNCS 5524: pp. 354-361, 2009.

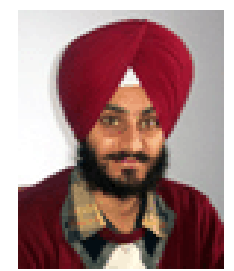

Mandeep Singh is an Asst. Prof at Thapar University, Patiala (INDIA) since 2003. He has done his M.Tech. in Instrumentation Engg. From Panjaab University, Chandigarh. He is pursuing his Ph.D. from Thapar University Patiala. His current research interests are Medical Imaging, Bio-Medical Instrumentation, Embedded systems and Signal processing. He is a member of IEEE and IACSIT. 